\title{
Major causes of death: spotlight on mTOR pathway
}

\begin{abstract}
Diseases of aging constitute the greatest proportion of major causes of death worldwide. mTOR pathway, well-known as a key player in the process of aging in human and many other species, has been shown to have a role in many of the diseases of aging. Here we review the role of mTOR pathway in several diseases of aging that are also established as major causes of death, to speculate the centrality of this pathway in human mortality.
\end{abstract}

Keywords: aging, causes of death, hyper function, mortality, mtor, hyper function, ros, neurodegenerative diseases, acquired immunodeficiency syndrome, chronic obstructive pulmonary disease, nutrient, theory of aging, damage accumulation theory, diseases of aging, ischemic heart disease
Volume 4 Issue I - 2017

\author{
Mohammad Farahmandnia, ${ }^{1,2}$ Mohammad \\ hossein Khorraminejad-Shirazi, ${ }^{1,2}$ \\ Mohammadreza Dorvash ${ }^{2,3}$ \\ 'School of Medicine, Shiraz University of Medical Science, Iran \\ ${ }^{2}$ Cell \& Molecular Medicine Student Research Lab, Shiraz \\ University of Medical Science, Iran \\ ${ }^{3}$ School of Pharmacy, Shiraz University of Medical Science, Iran
}

Correspondence: Mohammad Farahmandnia, Cell \& Molecular Medicine Research Lab, School of Medicine, Imam-Hossein Square, Shiraz, Fars, Iran, Tel +98937/240I44,

Email farzamfarahmandnia@gmail.com

Received: July 26, 2016 | Published: February 23, 2017
Abbreviations: AIDS, acquired immunodeficiency syndrome; COPD, chronic obstructive pulmonary disease; mTOR, mechanistic target of rapamycin; ROS, reactive oxygen species

\section{Introduction}

As tangible is aging to the human perception, it has proved hard to define. Some define aging as a decline in the function of different organs of the body, ${ }^{1}$ whereas some others define it as the hyper function of those organs. ${ }^{2}$ Whatever the definition of aging, it is well established that aging does correlate with the onset of many diseases that are also known as major causes of death worldwide. ${ }^{3}$ According to global report by WHO, top 10 causes of death as of 2012 are ischemic heart disease, stroke, chronic obstructive pulmonary disease (COPD), lower respiratory infections, cancers of respiratory system, acquired immunodeficiency syndrome (AIDS), diarrheal diseases, diabetes mellitus, road injury, and hypertensive heart disease, in the order of the number of deaths they are responsible for. ${ }^{4}$ (Figure 1) 6 out of these 10 are directly related to the process of aging, hence can be categorized under "diseases of aging", cumulatively accounting for $75 \%$ of deaths due to the top 10 causes, with the other 4 diseases only to blame for $25 \%$ of the mortalities (Figure 2). The above statistics mandate the need for a well-structured theory to explain how aging gives rise to fatal diseases that are currently the prime suspect for death worldwide. Recent theoretical works, alongside the growing body of evidence, implicate the relevance of hyper function theory of aging, while the damage accumulation theory falls short in rationally modeling the mechanism by which reactive oxygen species (ROS) trigger all of the diseases of aging. ${ }^{1,2,5}$ The hyper function theory is centered on a molecular pathway named mechanistic target of rapamycin (mTOR) pathway. This pathway functions as a relay that senses nutrient inputs and drives metabolic balance ${ }^{6}$ in cells, and it has been well established to have a remarkable role in diseases of aging ${ }^{7}$ most notably vascular accidents such as ischemic heart disease ${ }^{8}$ and stroke $^{9}$ cancers $^{7}$ diabetes mellitus ${ }^{7}$ and neurodegenerative diseases. ${ }^{10}$ This theory states that the intracellular processes involved in growth that occur in concert with the cell divisions during development, continue their course post-development when cell divisions are extensively limited. This continuation of growth, quasi-programmed by the processes that drive development, puts cells in a hyper function state without the ability to divide and reach balance again, hence resulting in the aging phenotype of the organs of the body. ${ }^{1-3}$

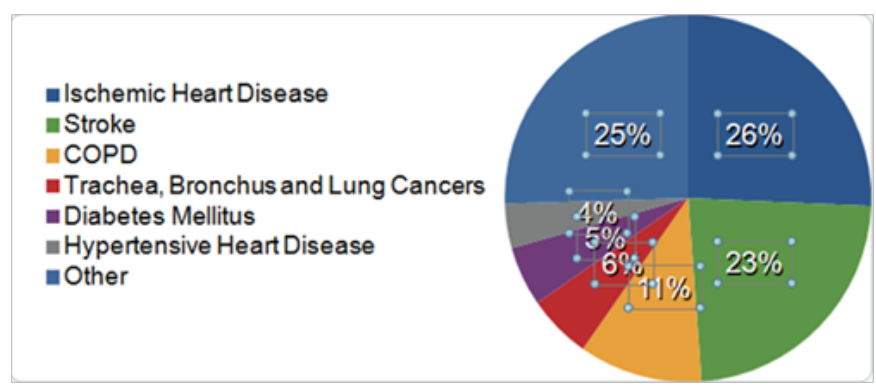

Figure I 6 out of 10 major causes of death strongly correlated with aging and their share of deaths in 2012 .

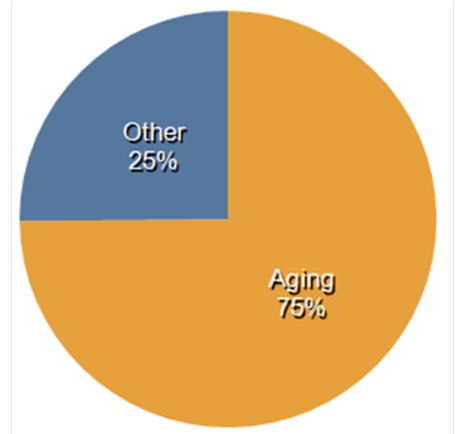

Figure 2 when calculated cumulatively, $75 \%$ of deaths caused by major causes are to blame on diseases of aging.

\section{Discussion}

Through hyper function theory of aging, the mechanisms that give rise to the most devastating diseases of aging can be explained. (Table 1) In light of this theory, it can be understood that these diseases are not distinct situations, but organ-specific presentations of one disease, aging. Almost all vascular accidents happen due to hypo perfusion/ ischemia, secondary to stenosis of the arteries of the involved organ. Stenosis is merely the result of growth of cells in the arterial wall. 
Hyper function of mTOR pathway has been shown as the mechanism that causes the increase in size in these cells. ${ }^{89}$ In tumorigenesis, cells manage to escape from the mechanisms that keep them in the hyper function state without permitting them to divide, thence reproducing hastily thanks to the hyperactivity of mTOR pathway that provides enough nutrients for them to utilize. ${ }^{7}$ Furthermore, hyper function of mTOR pathway in the vasculature surrounding the tumor, triggers angiogenesis, delivering evens more nutrients to the tumor. ${ }^{11}$ In diabetes mellitus, hyper function of mTOR pathway results in negative feedback build-up that prevents conduction of the signal of surface insulin receptors to the cell, resulting in insulin resistance. ${ }^{7}$ In Alzheimer's disease, hyper function of mTOR pathway causes increased protein synthesis, among which protein aggregates that make up amyloid beta plaques. ${ }^{10}$ The role of mTOR pathway has been implicated in other diseases of aging, such as osteoporosis, ${ }^{12}$ COPD,${ }^{13}$ and even skin wrinkles. ${ }^{14}$ In consequence, the hyper function of mTOR pathway is in fact the most important characteristic that all the diseases of aging have in common, which turns the spotlight on this pathway as the key modulator of the process of aging and all of the diseases derived from it.

Table I The mechanisms by which diseases of aging develop can be justified through mTOR pathway.

\begin{tabular}{ll}
\hline & Mechanism of disease development through mTOR pathway \\
\hline Vascular Accidents & Narrowing of arterial lumen due to hypertrophy of arterial wall ${ }^{8,9}$ \\
Cancers & Drives growth and invasiveness of tumors, ${ }^{7}$ Angiogenesis ${ }^{11}$ \\
Diabetes Mellitus & Dysregulated glucose intake and metabolism due to resistance \\
Neurodegenerative Diseases & Increased precursor protein production results in plaque formation ${ }^{10}$ \\
\hline
\end{tabular}

\section{Conclusion}

According to the report conducted by WHO, 6 out of 10 leading causes of death in the world in the year 2012, which could be categorized under the label "diseases of aging", account for $38.3 \%$ and 21400000 of lives lost in that year. ${ }^{4}$ Another report by U.S. NCHS, names 5 leading causes of death, 4 of which could also be named as diseases of aging. ${ }^{15}$ Point of argument, if one sums up the percentage of deaths caused by aging rather than calculating them disease by disease, it would be rather obvious that in our century, the leading cause of death worldwide is "aging".

Acknowledging mTOR pathway as the central controller of the process of aging necessitates deeper understanding of this pathway and its constituents, and investment in drug design projects that target this pathway, to prevent aging, decrease its lethal effects on the human community and ultimately, practically stop it.

\section{Acknowledgements}

None.

\section{Conflict of interest}

The author declares no conflict of interest.

\section{References}

1. Iseghohi, Sylvia Oghogho, Kingsley Omage. How ageing increases cancer susceptibility: A tale of two opposing yet synergistic views. Genes \& Diseases. 2016;3(2):105-109.

2. Blagosklonny MV. Aging: ROS or TOR. Cell Cycle. 2008;7(21):33443354 .

3. Blagosklonny MV. Rapamycin extends life-and health span because it slows aging. Aging (Albany NY). 2013;5(8):592-598.
4. http://www.who.int/mediacentre/factsheets/fs310/en/.

5. Zhu, Chunpeng, Wei Hu, et al. No evident dose-response relationship between cellular ROS level and its cytotoxicity-a paradoxical issue in ROS-based cancer therapy. Scientific reports 4. 2014.

6. Kim DH, Sarbassov DD, Ali SM, et al. mTOR interacts with raptor to form a nutrient-sensitive complex that signals to the cell growth machinery. Cell. 2002;110(2):163-175.

7. Zoncu R, Efeyan A, Sabatini DM. "mTOR: from growth signal integration to cancer, diabetes and ageing. Nat Rev Mol Cell Biol. 2011;12(1):21-35.

8. Lüscher TF, Steffel J, Eberli FR, et al. Drug-eluting stent and coronary thrombosis biological mechanisms and clinical implications. Circulation. 2007;115(8):1051-1058.

9. Xiong X, Xie R, Zhang H, et al. PRAS40 plays a pivotal role in protecting against stroke by linking the Akt and mTOR pathways. Neurobiol Dis. 2014;66:43-52.

10. Spilman P, Podlutskaya N, Hart MJ, et al. Inhibition of mTOR by rapamycin abolishes cognitive deficits and reduces amyloid-beta levels in a mouse model of Alzheimer's disease. PLoS One. 2010;5(4):e9979.

11. Karar J, Maity A. PI3K/AKT/mTOR pathway in Angiogenesis. Front Mol Neurosci. 2011;4:51.

12. Hadji P, Coleman R, Gnant M. Bone effects of mammalian target of rapamycin (mTOR) inhibition with everolimus. Crit Rev Oncol Hematol. 2013;87(2):101-111.

13. Mercado N, Ito K, Barnes PJ. Accelerated ageing of the lung in COPD: new concepts. Thorax thoraxjnl-2014. 2015;70(5):482-489.

14. Murakami H, Shimbo K, Inoue $\mathrm{Y}$, et al. Importance of amino acid composition to improve skin collagen protein synthesis rates in UVirradiated mice. Amino Acids. 2012;42(6):2481-2489.

15. Miniño AM, Murphy SL. Death in the United States, 2010. NCHS data brief, no 99.2012. 